\title{
MOTIVOS PARA EL DISEÑO DEL EXAMEN FINAL DE OCTAVO SEMESTRE DE LOS ESTUDIANTES DE LA FACULTAD DE ENFERMERÍA.
}

\author{
REASONS FOR THE DESIGN OF THE FINAL EXAM OF 8TH SEMESTER \\ STUDENTS OF THE SCHOOL OF NURSING
}

Josefina Valenzuela Gandarilla

Maestra en Educación Médica. Profesor e investigador, adscrita a la Facultad de Enfermería de la Universidad Michoacana de San Nicolás de Hidalgo. josefina_vgandarilla@yahoo.com.mx

David Mendoza Armas Dr. En Ciencias en Ciencias de la Educación. Facilitador del doctorado en la Universidad Autónoma de Durango. drmendozaarmas@live.com.mx

María Dolores Flores Solís

Maestra en Educación Médica. Profesor e investigador, adscrita a la Facultad de Enfermería de la Universidad Michoacana de San Nicolás de Hidalgo. doloresfloresnut@hotmail.com

Elizabeth Medina Castro Dra. En Ciencias en Enfermería. Profesor e investigador, adscrita a la Facultad de Enfermería de la Universidad Michoacana de San Nicolás de Hidalgo. elizabeth_lizma@hotmail.com

\section{Resumen}

En el presente estudio el objetivo fue determinar los motivos que originaron el diseño del examen final para alumnos de octavo de la Facultad de Enfermería de la Universidad Michoacana San Nicolás de Hidalgo de Morelia, Michoacán. Se trató de un estudio de desarrollo en el campo de la evaluación. Resultados: los estudiantes tenían entre 21 - 23 años en un $77.5 \%$, el $88.7 \%$ fueron mujeres. Los informantes clave tenían entre 5 y 39 años como docente. El $87.3 \%$ expresó que sí, que un examen final podrá contribuir para el desempeño de enfermería. Esto indica que las y los estudiantes consideran que es necesaria una evaluación final. Asimismo se 
podrán medir conocimientos e identificar de deficiencias en la formación profesional.

Palabras clave: desempeño, examen final, evaluación, formación profesional, motivos.

\begin{abstract}
In the present study aimed to determine the reasons for the design of the final exam semester eigth students at the School of Nursing at the Universidad Michoacana San Nicolas de Hidalgo in Morelia, Michoacan. It was a development studio in the field of evaluation. Results: The students were between 21 to 23 years $77.5 \%, 88.7 \%$ were women. Key informants were between 5 and 39 years as a teacher. $87.3 \%$ expressed that if, a final exam may contribute for the performance of nursing. This suggests that students consider and the need for a final evaluation. Also be measurable knowledge and identify gaps in training.
\end{abstract}

Keywords: performance, final examination, evaluation, training, reasons.

\title{
Introducción
}

La educación superior para el siglo XXI debe asumir el cambio y el futuro como consustanciales de su ser y quehacer (Delors, 1996). El futuro exige de las instituciones de educación superior una predisposición a la reforma constante de sus estructuras y métodos de trabajo, lo cual implica asumir la flexibilidad como norma de trabajo, en lugar de la rigidez y el apego a tradiciones inmutables. A su vez, la instalación en el futuro y la incorporación de la visión prospectiva en su labor, hará que la educación superior contribuya a la elaboración de los proyectos futuros de la sociedad, inspirados en la solidaridad, en la superación de las desigualdades y en el respeto al ambiente. La agenda de Compromiso "Libertad creadora y desarrollo humano en una cultura de paz", aprobada por aclamación en la "Reunión Internacional de Reflexión sobre los Nuevos Roles de la Educación Superior", convocada por la UNESCO (Caracas, mayo de 1991), reconoció como el mayor reto de la educación superior en los próximos años su contribución a "la construcción y redefinición de un nuevo pensamiento capaz de identificar los términos de un 
proyecto social compatible con las exigencias que derivan de la necesidad de superar las marcadas desigualdades sociales, integrando a los pueblos como actores de su propia legitimidad" (Conferencia Mundial; 1988).

Desde esta perspectiva la formación de los docentes es un punto clave para tener logros en la educación superior y en especial en la gestión docente. Dirigir el proceso docente-educativo en la actualidad implica tener en cuenta un enfoque sistémico, considerando a los objetivos o aspiraciones que deben ser logrados por los estudiantes como un elemento rector. En este proceso, la evaluación tiene un importante papel relacionado con la necesaria retroalimentación de la gestión desarrollada en relación con la formación del que aprende, este enfoque integral y sistémico de la enseñanza-aprendizaje se relaciona con las tendencias más actuales en esta área y de hecho constituyen un importante referente de conocimientos pertinentes, aspiración educacional que caracteriza a la educación superior. La educación Médica no es ajena a estas consideraciones y desde hace varias décadas viene planteando una serie de recomendaciones en el orden pedagógico en relación con una dirección docente pertinente al encargo social (Cumbre Mundial Edimburgo; 1993). Dentro de las funciones de la Universidad Michoacana de San Nicolás de Hidalgo se encuentra la de formar profesionales para que sirvan a su patria con eficiencia y rectitud (Ley Orgánica, 2004) de ahí la importancia de la evaluación que permita garantizar el cumplimiento de las funciones de esta máxima casa de estudios. En este sentido, la carrera de Licenciado en Enfermería viene dando sólidos pasos en México, aunque aún queda un gran camino por recorrer. En el Estado de Michoacán, ámbito de este estudio, la Facultad de Enfermería es la principal formadora de estos recursos y aunque al egreso el total de las y los estudiantes presentan el EGEL-ENFER de CENEVAL, en la Facultad no se cuenta con un examen final teórico-práctico para los estudiantes que están a punto de egresar de la carrera.

El plan de estudios, consta de ocho semestres, en los cuales los estudiantes adquieren los conocimientos para la atención directa a la persona, desde el cuidado general de la enfermería hasta el cuidado en especialidad como la salud pública, la salud reproductiva, enfermería quirúrgica, enfermería médico-quirúrgica, salud mental y la administración y gestión de recursos humanos. El mapa curricular 
contempla disciplinas cuya información y formación se relacionan con las funciones de atención directa, docencia, administración e investigación. Incluye cinco líneas de formación: 1) El hombre y su ambiente, 2) Ciencias biomédicas, 3) Docencia e investigación, 4) Sociedad y salud, y; 5) Comunicación y lenguaje. Es en los profesores que imparten las diversas materias del área de enfermería y en los estudiantes de octavo semestre de este plan de estudios en los cuales se realizó la presente investigación.

En el octavo semestre el estudiante está en una etapa preprofesional, momento donde debe mostrar el desarrollo de las competencias profesionales adquiridas en función del desempeño esperado. Es importante enfatizar en la importancia que tiene la evaluación de los conocimientos y habilidades profesionales con las que cuentan los estudiantes que se encuentran "a un paso" de incorporarse a la práctica pre-profesional, ya que tal constatación garantiza tener importantes elementos acerca del logro de los objetivos previstos en la carrera, lo que al mismo tiempo contribuye a que la institución educativa, cuente con una información objetiva acerca de si el futuro profesional está formado para ser resolutivo en el ámbito en el cual va a desempeñarse, pues cada vez más se aspira a un comportamiento de mayor calidad en relación con la práctica profesional de enfermería. Teniendo en cuenta lo valorado con anterioridad, no se cuenta con ninguna investigación en la cual se hayan determinado los motivos para el diseño de un examen final de octavo semestre, que permita la constatación del logro de los sistemas de conocimientos y habilidades profesionales, previstos en el perfil del egresado en respuesta al encargo social de este contexto, en relación con el cuidado de enfermería que poseen los estudiantes a su egreso, por ello el desarrollo de un proceso investigativo en esta área contribuye a la necesaria profundización en la misma con un enfoque contextualizado dado que la institución educativa contará con importantes resultados acerca de cómo está garantizando a la sociedad un profesional formado con las competencias profesionales, generales y específicas que se requieren para ser resolutivos a las necesidades sociales que se exigen.

\section{Desarrollo}


El aprendizaje es un proceso cíclico y en espiral que continúa y cambia a través del tiempo y su función transformadora exige del profesor una evaluación continua y objetiva. El aprendizaje abarca un proceso de equilibrio dinámico entre: la estabilidad y el cambio, la estructura y el proceso, y; el contenido y la actividad. La información orientada es un factor clave en el aprendizaje: no se puede avanzar ni dar un salto cualitativo en el comportamiento profesional, sino se cuenta con informaciones que permitan ampliar la conciencia en torno a un problema. En la medida en que el aprendizaje ocurre se desarrollan organizaciones del conocimiento bien estructuradas y cualitativamente diferentes, que capacitan los aprendices para construir una representación compleja o modelo mental que los guía tanto en la solución de problemas como en su aprendizaje futuro. (González, 2009).

El proceso de concientización debe pasar de una fase inicial cargada de interrogantes y contradicciones a una etapa cada vez más plena, donde se profundiza el estudio del problema y se le comprende en su totalidad. Esta es una de las cuestiones claves de la llamada "didáctica de la problematización" (Rivera, 2004). Una cuestión básica relacionada con el aprendizaje es la motivación. Quien no necesite aprender es imposible que aprenda. (Fermoso, 2009).

En determinados momentos la información se "suministra" a un educando que se ha convertido en un sujeto activo (Salas, 1998) que participa en su propia formación; mientras que con frecuencia es el propio educando quien se moviliza en la búsqueda de la información, todo lo cual tiene lugar en la interacción profesor-alumno como una relación de la posición orientadora del que enseña y la posición productiva del que aprende. En ello juega un papel determinante la motivación y la afectividad del propio estudiante, que debe ser estimulada desde el comienzo del proceso docente en la medida en que el sujeto se siente envuelto y protagonista de los desafíos del aprendizaje. Es en este contexto, donde la evaluación evidencia su mayor fuerza en la función instructiva y educativa que la caracteriza (Sackett, 2001).

En este enfoque formativo, los estudiantes se convierten en el soporte y fuente principal de su aprendizaje, mientras que el profesor es más un estimulador, orientador y catalizador, que un instructor en sí mismo. Los alumnos por tanto son vistos como alguien inmerso en 
un proceso de formación, que le permitirá al final contar con conocimientos, habilidades, conductas y valores a través del proceso educacional, donde resulta fundamental tener una clara visión evaluativa. Desde esta perspectiva, los profesores son facilitadores de todo el proceso educacional, tanto como organizadores y ejecutores del proceso, así como también controladores y evaluadores de sus resultados, los que se reflejarán en su dimensión más general, una vez que los estudiantes egresen y se incorporen al mercado laboral y con su capacidad resolutiva logren un impacto positivo en la población que atiendan. Por ello la planificación y ejecución de las evaluaciones de carácter certificativo desempeñan un papel fundamental (Salas, 1999), lo que se hace particularmente importante en aquellas relacionadas con los espacios formativos del final de la carrera de Licenciado en Enfermería. Los profesores, que asumen como función esencial la orientación desarrollan el proceso educativo basado en la combinación acertada de los métodos de enseñanza, cada vez más encaminados al aprendizaje basado en la solución de problemas, lo cual exige de la relación educativa horizontal, el fortalecimiento del trabajo grupal, la reivindicación del diálogo como parte del proceso de adquisición de los conocimientos, la identificación del contexto principal o estudio de un problema derivado de la realidad, y la adquisición de conocimientos dentro de un proceso progresivo y dinámico. De tal forma que las escuelas y facultades del área de la salud o ciencias médicas deben contribuir a promover, conservar y restaurar la salud del hombre, teniendo siempre en cuenta un marco de referencia humanista. Contribuyendo a mejorar con sus acciones la salud de la población estableciendo así un compromiso con el desarrollo científico, social, ecológico y humanístico de cada comunidad, región o país atendiendo a las peculiaridades y circunstancias de cada uno (Pacheco, 1990).

La personalidad profesional que exige la sociedad a la Educación superior no nace con el ser humano, sino que su desarrollo constituye una consecuencia del proceso de asimilación de la experiencia histórico social (Rivera, 2004). Por ello se debe tener en cuenta la relación social entre los hombres y el tipo de actividad que en la interrelación se lleva a cabo para lograr las transformaciones cualitativas que la sociedad reclama en el tipo de profesional que exige. Al considerar desde este enfoque el esencial papel transformador y desarrollador que tiene la educación para el ser 
humano (Rivera, Dereck; 2004), se puede comprender la importancia de este proceso en la formación profesional y en particular en la formación de los recursos humanos en salud. Resulta importante el papel que tiene el profesor como máximo responsable en la formación de un recurso humano que tiene que ser portador no sólo de elementos científicos y técnicos sino también de valores morales, proceso formativo donde la evaluación juega un papel fundamental (Salas, 2005).

\section{Metodología}

Se trató de un estudio de desarrollo, en el campo de la evaluación, en el cual se determinaron los motivos para diseñar el examen final de octavo semestre a los estudiantes de la Facultad de enfermería de la Universidad Michoacana de San Nicolás de Hidalgo. Se hizo uso de los modelos de investigación cualitativos y cuantitativos, el sistema de métodos utilizados integró esencialmente a los teóricos y empíricos, donde se consideró la encuesta y la entrevista, esto permitió la obtención de precisiones significativas sobre aspectos trascendentales del proceso evaluativo y educativo en general de la carrera objeto de estudio. Los métodos teóricos permitieron el análisis documental de la literatura especializada sobre el tema, de enfermerías y otras fuentes de interés. Se aplicaron dos instrumentos, uno a 71 estudiantes que constó de 12 preguntas de las cuales siete fueron mixtas, tres cerradas y dos abiertas. El segundo instrumento constó de nueve preguntas abiertas y se aplicó a 15 profesores que impartían las asignaturas de enfermería en las diferentes áreas, ya que es ésta el eje integrador de la carrera. Los instrumentos fueron validados por criterio de expertos. El muestreo fue aleatorio por conveniencia, estando compuesta por 71 estudiantes que cursaban el octavo semestre y 15 profesores que impartían la materia de enfermería. La confidencialidad de los resultados estuvo garantizada por la ética de estudio y se hizo uso del paquete estadístico SPSS V.15.

\section{Resultados}

La edad de los estudiantes encuestados en el $77.5 \%$ tenía entre 21 23 años, el 15.5 entre 24 - 26 años, con relación al género, el 88.7\% fueron mujeres. Al cuestionar acerca de si el diseño de un examen 
final podrá favorecer el desempeño de enfermería, el 87.3\% expresó que si y el $12.7 \%$ que no. Esto indica que las y los estudiantes consideran que una evaluación final con un instrumento bien estructurado si favorecería su desempeño una vez que egrese. Al indagar acerca del porqué se favorecería el desempeño, las respuestas fueron que con este tipo de exámenes se medirían los conocimientos que poseen y se identificarían deficiencias. Los resultados obtenidos en este sentido fueron de gran utilidad toda vez que más de las $3 / 4$ partes de los encuestados tienen claro que con un examen final que mida conocimientos y habilidades se contribuiría en mayor medida al logro del desempeño que en el ámbito laboral se espera de ellos.

Con relación a los informantes clave, éstos tenían una antigüedad de entre 5 y 39 años de servicio y más de las $2 / 3$ partes de éstos tenían más de 20 años dedicándose a la docencia, impartían las diferentes materias de enfermería. Al indagar acerca de si los exámenes finales debían dirigirse a constatar las posibilidades de aplicación del contenido por parte del estudiante, más de la tercera parte de las encuestados refirió que sí porque de esa manera se constata si los alumnos en realidad saben y pueden aplicar los contenidos en la práctica; además de que demuestran si poseen los conocimientos en el momento en que los necesiten para solucionar los problemas que se les presente. Al indagar sobre el valor que se atribuye a constatar la aplicación por parte del estudiante en el octavo semestre, más de las dos terceras partes de los informantes clave refirió que sería bueno ya que se valoraría la formación integral entre ellas las actitudes, la capacidad de discernir y aplicar los conocimientos, pero que se deben proporcionar los elementos teórico-metodológicos para que los estudiantes continúen desarrollándose por sí mismos. De igual forma hacen referencia a que permitiría valorar el proceso de enseñanza y aprendizaje de manera íntegra. Estos argumentos coinciden con la bibliografía consultada y con el criterio de la propia autora.

Al indagar sobre el diseño de un examen final que mida conocimientos y habilidades en los estudiantes más de la mitad de los encuestados refirieron que sería muy bueno ya que permitiría evaluar la calidad profesional real con la que egresan los alumnos, identificar áreas débiles y determinar estrategias para reforzarlas, de la misma 
manera se tendría un panorama de que pasó durante la carrera teniendo un diagnóstico de la permanencia del alumno. Estos argumentos coinciden con lo expresado por los estudiantes y con los criterios asumidos por los propios investigadores.

Respecto a la modalidad que debería tener el examen final, prácticamente el total de las encuestadas consideró que éste debe ser teórico práctico, con estudios de casos y casos clínicos que permitan explorar en los estudiantes no sólo la memorización sino su actuación en casos específicos.

Respecto a los contenidos que deben incluirse en el examen final, prácticamente el total de los docentes entrevistados considera que deben ser desde la perspectiva de la enfermería ya que es el eje integrador de la carrera, existe concordancia con los resultados obtenidos de los estudiantes quienes refirieron que las preguntas, debían dirigirse a explorar no sólo conocimientos sino también aspectos relacionados con la práctica de enfermería, otras respuestas a tener en cuenta plantearon la importancia de las preguntas de complementación, selección múltiple y también de solución de casos problemáticos. De acuerdo a la literatura consultada los tipos de preguntas deben pensarse considerando su combinación según los requerimientos de los objetivos a lograr y que deberán ser constatados para que permita a los estudiantes expresar sus pensamientos y no encasillarlos sólo en opciones. Los autores opinan, que de acuerdo a la experiencia docente con que se cuenta, a las materias de enfermería, tributan el resto y con la estructuración de un examen válido y confiable se medirían las competencias y el desempeño del estudiante.

\section{Conclusión}

Los argumento expresados por los estudiantes e informantes clave coinciden y se constituyen en criterios bien definidos en las entrevistas realizadas y ratificaron la pertinencia del estudio, en relación con los motivos para el diseño de examen final para el octavo semestre que explorará las competencias y el desempeño que deben caracterizar al Licenciado en Enfermería. 
Los motivos que se mencionaron para el diseño del examen final de octavo semestre fueron evaluar la calidad profesional real con la que egresan los alumnos, identificar áreas débiles y determinar estrategias para reforzarlas. Así como el panorama durante la carrera, logrando con ello un diagnóstico de la permanencia del alumno.

Las coincidencias encontradas relacionadas con la necesidad del mismo y la incorporación de una modalidad que considere la práctica de enfermería fueron criterios planteados por los sujetos estudiados y corroborados de manera general con la bibliografía consultada en relación con experiencias de otras universidades y de México que asumen la carrera de Licenciado en Enfermería.

\section{FUENTES DE CONSULTA}

- BORROTO RE. (2004) Pertinencia de la educación superior en el siglo XXI por Michael Gibbons. Maestría en Educación Médica: La Habana.

- Conferencia Mundial Sobre La Educación Superior (1998) UNESCO, París 5-9 de octubre.

- Cumbre Mundial sobre Educación Médica (1993) La Profesión Médica en Evolución. Recomendaciones: Edimburgo.

- DELORS, J. (1996) La Educación Encierra un Tesoro Informe de la UNESCO de la comisión Internacional sobre la educación para el siglo XXI: Santillana, Madrid.

- DÍAZ, F. (2003) Cognición situada y estrategias para el aprendizaje significativo. Revista Electrónica de Investigación Educativa, 5 (2). Consultado el 25 de noviembre de 2011 en: http://redie.ens.uabc.mx/vol5no2/contenido-arceo.html.

- FERMOSO, E.P. (2009). Teorías de la educación. Ed. Trillas. México, D.F.

- GONZÁLEZ, L.D. (2009). Estrategias referidas al aprendizaje, la instrucción y la evaluación. Ed. Pearson. México. D.F. 
- Ley Orgánica (2004) Legislación Universitaria I. Normas jurídicas fundamentales. Estatuto universitario.

- Plan de Estudios (1998) Escuela de Licenciatura en Enfermería de la Universidad Michoacana de San Nicolás de Hidalgo. Morelia, Michoacán. 1998.

- Plan de estudios (2008) Facultad de Enfermería de la Universidad Michoacana de San Nicolás de Hidalgo.

- NOGUEIRA M. y RIVERA N. (2003) Desarrollo de competencias para la gestión en la educación médica superior de la Habana. En: Revista de educación Médica; Vol 3. La Habana.

- MARRINEY -T. (2004) Modelos y teorías de enfermería. Ed. Doyma. España.

- IRIGOIN M. y Vargas. F. (2002) Competencia laboral . OPS./CINTERFOR.

- Marco Jurídico (1995) Universidad Michoacana de San Nicolás de Hidalgo. Morelia, Michoacán.

- FERNÁNDEZ, J.A. (2004) Educación Médica Superior, realidades y perspectivas a las puertas del nuevo siglo. Universidad Médica de la Habana.

- PIAGET, Jean. (1997) Estudios de psicología genética. Emecé editores.

- RIVERA, N. (2004) Proceso enseñanza aprendizaje: Lecturas seleccionadas. Maestría de Educación Médica. La Habana, Cuba.

- RIVERA, N. (2004) Enfoque psicopedagógico del proceso docente: Su impacto en el desarrollo de la personalidad. Maestría en Educación Médica: La Habana, Cuba.

- SALAS, R. (1998) La evaluación en la educación superior contemporánea. Biblioteca de medicina Volumen XXIV. Capítulo 5. Universidad Mayor de San Andrés, La Paz. 
